\title{
Brief FASD prevention intervention: physicians' skills demonstrated in a clinical trial in Russia
}

Tatiana Balachova ${ }^{1 *}$, Barbara L Bonner ${ }^{1}$, Mark Chaffin ${ }^{1}$, Galina Isurina ${ }^{2}$, Vladimir Shapkaitz ${ }^{3}$, Larissa Tsvetkova², Elena Volkova ${ }^{4}$, Irina Grandilevskaya ${ }^{2}$, Larissa Skitnevskaya ${ }^{4}$ and Nicholas Knowlton ${ }^{5}$

\begin{abstract}
Background: Alcohol consumption during pregnancy can result in a range of adverse pregnancy outcomes including Fetal Alcohol Spectrum Disorders (FASD). Risky drinking among Russian women constitutes a significant risk for alcohol-exposed pregnancies (AEP). Russian women report that obstetrics and gynecology (OB/GYN) physicians are the most important source of information about alcohol consumption during pregnancy and developing effective prevention interventions by OB/GYNs is indicated. This is the first study focused on implementation of an AEP prevention intervention at women's clinics in Russia.

Method: The paper describes the intervention protocol and addresses questions about the feasibility of a brief FASD prevention intervention delivered by OB/GYNs at women's clinics in Russia. Brief physician intervention guidelines and two evidence-based FASD prevention interventions were utilized to design a brief dual-focused physician intervention (DFBPI) appropriate to Russian OB/GYN care. The questions answered were whether trained OB/GYN physicians could deliver DFBPI during women's routine clinic visits, whether they maintained skills over time in clinical settings, and which specific intervention components were better maintained. Data were collected as part of a larger study aimed at evaluating effectiveness of DFBPI in reducing AEP risk in non-pregnant women. Methods of monitoring the intervention delivery included fidelity check lists (FCL) with the key components of the intervention completed by physicians and patients and live and audio taped observations of intervention sessions. Physicians ( $N=23$ ) and women ( $N=372$ ) independently completed $F C L$, and 78 audiotapes were coded.
\end{abstract}

Results: The differences between women's and physicians' reports on individual items were not significant. Although the majority of physician and patient reports were consistent $(N=305)$, a discrepancy existed between the reports in 57 cases. Women reported more intervention components missing compared to physicians $(p<0.001)$. Discussing barriers was the most difficult component for physicians to implement, and OB/GYN demonstrated difficulties in discussing contraception methods.

Conclusions: The results supported the feasibility of the DFBPI in Russia. OB/GYN physicians trained in the DFBPI, monitored, and supported were able to implement and maintain skills during the study. In addition to the alcohol focus, DFBPI training needs to have a sufficient component to improve physicians' skills in discussing contraception use.

\section{Background}

Alcohol use during pregnancy is the leading preventable cause of mental retardation and can result in Fetal Alcohol Syndrome (FAS) and a wide range of Fetal Alcohol Spectrum Disorders (FASD) [1-4]. The worldwide rate of FAS has been estimated to be 1.9 per 1,000 live births [5]. Recent studies indicate a higher FAS rate of 2 to 7 per

\footnotetext{
*Correspondence: Tatiana-Balachova@ouhsc.edu

${ }^{1}$ The University of Oklahoma Health Sciences Center, 940 N.E. 13th Street, Nicholson Tower Suite 4900, Oklahoma City, OK 73104, USA

Full list of author information is available at the end of the article
}

1,000 in the US, and FASD prevalence is estimated to be $2 \%-5 \%$ among elementary school children in the US and some Western European countries [6]. The rates are likely to be higher in countries with greater alcohol use and limited education about the effects of alcohol consumption during pregnancy. Although the FAS rates in Russian general populations have not been established, studies report high FAS and FASD rates in Russian orphanages [7-9] and in children adopted from Russia [10].

FAS and FASD are completely preventable by avoiding alcohol use during pregnancy [11,12]. Approximately $12 \%$

\section{Biomed Central}


of women in the US [13] and over 20\% worldwide consume alcohol during pregnancy [14]. Most women eliminate or reduce alcohol consumption on learning that they are pregnant. However, approximately half of all pregnancies are unplanned, and many women are not aware they are pregnant until four to six weeks into pregnancy and continue using alcohol at pre-pregnancy levels [15]. As a result, a significant proportion of women consume alcohol during the early stages of pregnancy prior to pregnancy identification $[14,16]$. Studies indicate that alcohol exposure early in pregnancy may affect fetal development even if followed by later gestational abstinence [17]. A combination of at-risk drinking with the possibility of becoming pregnant constitutes a significant risk for alcohol-exposed pregnancies (AEP), and a pre-conceptional approach to preventing FASD has been recommended [18].

In Russia, nearly all women report drinking in the year before pregnancy, and depending on the study, 20\%-60\% drink to some extent after pregnancy recognition, and $3 \%-7.4 \%$ report binge drinking during pregnancy $[19,20]$. In a longitudinal outcome study of 413 pregnant women in Moscow, 20.2\% reported binge drinking around the time of conception, and $4.8 \%$ reported binge drinking in the most recent month of pregnancy [21]. In a sample of 648 women recruited from women's clinics in two regions of Russia, between $32 \%$ and $54 \%$ of non-pregnant women were at risk for $\mathrm{AEP}^{\mathrm{a}}$ [19] in contrast to $2 \%$ of non-pregnant women in a US national sample [22]. Applying comparable risk criteria, the general population at-risk rate in Russia (32\%-54\%) was higher then that of the highest risk women in the US, i.e., US women at drug and alcohol treatment centers US (24\%) [22]. Many Russian women eliminate or significantly curtail alcohol consumption after pregnancy recognition, but minimal reduction in use occurs during the pre-conception period, even among women who are actively attempting to become pregnant [19]. Among women who were trying to conceive, $67 \%$ reported binge drinking in the prior three months [14]. The prevalence of binge drinking among Russian women who might become or are trying to become pregnant constitutes a significant public health problem.

Brief physician intervention (BPI) has been recognized as an effective approach to reducing alcohol use and related health problems in patients at risk in primary care [23,24]. Although research provides some evidence that BPI reduces women's AEP risk [25-32], studies are needed to ascertain the efficacy of brief interventions (BI) for women and to determine the type of AEP prevention interventions that could be the most effectively implemented in primary health care [33-35]. BPI can be effective in reducing AEP risk if it is feasible, deliverable, and correctly implemented; however, feasibility has sometimes proven challenging [36-39]. In order to have a significant public health impact, intervention models must have both efficacy and deliv- erability. In other words, the intervention must be amenable to implementation, at scale, within authentic service systems, with reasonable fidelity and quality, and in substantial quantity. Feasibility of AEP prevention utilizing BPI in the Russian cultural context and health care system has not been studied.

Our initial studies of AEP risk in Russia used survey and interview methods with women and physicians in order to inform development of an AEP prevention strategy. Key findings included that a) the periconceptual period appeared to be a critical risk window; b) Russian women viewed their $\mathrm{OB} / \mathrm{GYN}$ physicians as having perhaps the single strongest influence on their health beliefs and behaviors; c) most women already modify their drinking after pregnancy recognition, largely due to an interest in their baby's health; and d) most women are unaware of AEP risk prior to pregnancy identification. Based on these key findings, we adapted a BPI model (to be described in detail later) and began implementing it at OB/GYN clinics in Russia. The parent study was a two-arm, 20-site, siterandomized trial aimed at evaluating the effectiveness of an intervention to reduce the risk for AEP in non-pregnant women. OB/GYNs ("women's clinics") were randomly assigned to intervention or control (no intervention) condition, and study participants were recruited from both intervention (10 clinics) and control sites (10 clinics). The aim of the current paper is to describe the BPI model that was developed and deployed at the intervention clinics, and present information drawn from implementation of quality control efforts about its feasibility and deliverability in the Russian context. This is the first study focused on implementation of an AEP prevention intervention at women's clinics in Russia. Subsequent papers will examine intervention impact on downstream client level AEP risk outcomes.

\section{Methods}

The study was reviewed and approved by Institutional Review Boards at St. Petersburg State University (SPSU) and the University of Oklahoma Health Sciences Center (OUHSC) and was conducted with approvals from the participating clinics.

\section{Setting and participants}

The study was conducted at public women's clinics in two locations in Russia represented by the major urban population of St. Petersburg (SPB) and more rural population of the Nizhny Novgorod Region (NNR). A total of ten clinics, five at each location (SPB and NNR), were assigned to the intervention. The clinics varied from a small rural clinic with one OB/GYN in the NNR to a large urban clinic with over $20 \mathrm{OB} / \mathrm{GYNs}$ in SPB. Commitments from the SPB and NNR Health Administrations were received to ensure cooperation from the participating clinic directors. 
Organizational support was obtained from the clinic directors to participate in the study and to allow participating physicians at intervention clinics to incorporate the study intervention into routine clinic visits with the study participants. Participating physicians met the following criteria: 1) certified in obstetrics and gynecology, 2) employed at least $50 \%$ time at a clinic assigned to the intervention, 3) agreed to serve in the study, 4) participated in the intervention training, and 5) demonstrated skills in completing the intervention protocol. A total of $26 \mathrm{OB} / \mathrm{GYN}$ physicians were trained in the protocol. Two did not commit to participate in the study, and one did not meet the post-training skills criteria and was removed. A total of $23 \mathrm{OB} / \mathrm{GYN}$ physicians (8 in NNR and 15 in SPB) participated in the study as interventionists. The physicians were female with a mean age of 38 years and average of 13 years in practice. Physicians were reimbursed approximately $\$ 20$ per intervention.

Patient participants were recruited for the study as consecutively enrolled non-pregnant women who were at risk for AEP between July, 2009-July, 2011. Patient inclusion criteria were: a) childbearing age women (ages 18-44 years); b) fertile; c) not currently pregnant (by self-report or a test result); d) engaging in AEP risk behaviors, i.e., specifically reporting having unprotected intercourse at least once in the last six months and drinking eight or more drinks per week on average or four or more drinks on one occasion within the past three months; e) living in the area served by one of the study clinics; f) available for follow-up for 12 months; and g) providing voluntary informed consent. A plan was made to over-recruit women with higher alcohol consumption to have at least $20 \%$ of the sample score 8 or higher on the Alcohol Use Disorders Identification Test (AUDIT) [40]. A review of women's AUDIT scores conducted after enrolling $80 \%$ of the targeted sample at each study location indicated a significant number of study participants with high AUDIT scores at the majority of clinics $(\mathrm{N}=8)$. At the remaining two clinics, AUDIT was administered upon screening to recruit few heavier drinkers. A total of 374 women were recruited at the 10 intervention clinics in SPB $(n=197)$ and the NNR $(n=177)$; $29 \%$ of the study participants scored 8 or higher on AUDIT. Participants received a gift at completion of the baseline assessment and the first intervention session (an equivalent of $\$ 25)$.

\section{Intervention protocol}

The intervention was adapted from two evidence-based FASD prevention interventions, Healthy Moms [30] and Project CHOICES [41]. Results from our previous studies in Russia guided selection and adaptation of this intervention protocol. The high prevalence of AEP risk among non-pregnant women in Russia who combine at-risk drinking with a possibility of becoming pregnant [19] dictated a need in intervention that would target women prior to pregnancy, e.g., non-pregnant women of childbearing age, and addressing both behaviors that place women at AEP risk, e.g. at-risk drinking and inconsistent family planning/ contraception. Women from our prior studies indicated that advice by OB/GYN physicians or nurses would be the most trusted source of information about health behaviors and alcohol consumption during pregnancy [42]. There is a well-established Russian OB/GYN health care system with services such as prenatal care and family planning/contraception services provided at district women's clinics free of charge. Based on our prior surveys of physicians and interviews with Russian experts, it was decided that the intervention protocol should be brief, incorporated in a routine clinic visit, and should require one to two sessions maximum as it is unlikely that some non-pregnant women would return for more than one follow-up visit. This is the first intervention protocol for AEP prevention in Russia and the first protocol for a dual-focused AEP prevention intervention designed to be deliverable by OB/GYN physicians during routine women's clinic visits.

First, we reviewed BI guidelines to make certain that the major components of effective interventions (e.g., advice, feedback, goal setting, additional contacts for further assistance, and support $[24,43]$ ) were included in the intervention protocol. Second, we reviewed FASD prevention studies and extracted elements from two evidence-based FASD prevention interventions with sound evidence for reducing AEP risk in non-pregnant women: Healthy Moms [30] and Project CHOICES [41]. Project CHOICES is a dual-focused intervention drawn from the Motivational Interviewing (MI) [44] framework and designed to decrease the AEP risk in non-pregnant childbearing age women by either reducing drinking or improving contraception or both. However, CHOICES itself could not be directly used within our intended parameters because it requires four 45 to $60 \mathrm{~min}$ counseling sessions with a mental health professional/counselor and one contraception session with a family planning clinician. A key reason for selecting CHOICES as one source for adaption to the Russian context was its flexibility in targeting both prevalent among Russian women problematic behaviors (risky drinking and lack of contraception) in one intervention. Also, OB/ GYN physicians assess and assist women in contraception use and are in a unique position to deliver this facet of the CHOICES approach. This is the first feasibility study testing delivery a dual-focused AEP prevention intervention by OB/GYN physicians.

We adapted the structural elements from the Healthy Moms [30] protocol to make our intervention deliverable during routine clinic visits. The Healthy Moms protocol was designed for women in the postpartum period to be deliverable in two 15-min clinic visits followed by two phone calls by OB/GYNs, outpatient nurses, or research staff. Similarly to CHOICES, Healthy Moms utilizes MI 
and includes a patient workbook that contains results of screening and personalized feedback about AEP risk, worksheets on drinking (and contraception in CHOICES), and drinking diary cards.

The adapted protocol, which was termed the DualFocused Brief Physician Intervention protocol (DFBPI), implements MI principles, focuses on both contraception and alcohol use, and is designed to be deliverable routinely by OB/GYN physicians at women's clinics. The intervention targets childbearing age non-pregnant women who are at risk for AEP, i.e., risky drinkers who are using contraception inconsistently. The DFBPI protocol and materials were prepared in consultation with Russian project consultants, obstetricians and behavioral health experts, including Russian women. The CHOICES and Healthy Moms intervention materials (e.g. workbooks) were translated and modified in accordance with DFBPI. Materials were translated and back translated by bi-lingual behavioral health experts in order to ensure that the materials were culturally congruent, accurate, and would be correctly comprehended by Russian women. A physician training protocol was developed and pre-tested by the study research group in a small randomized educational trial using a two-arm, pre/ posttest design [45]. The results of this study showed that Russian OB/GYN physicians randomized to the training condition demonstrated significantly improved skills after the training.

The DFBPI, with the translated title, Baby's Health is Your Choice, consisting of two face-to-face structured brief 5-10 min intervention sessions was incorporated in $\mathrm{OB} /$ GYN clinic visits scheduled approximately one month apart. Because of the lack of informational materials about contraception in Russia, an educational brochure [46] about contraception methods was also developed for the study. The DFBPI physician algorithm or steps to be taken by physicians are included in Figure 1.

In the intervention condition during the first visit, a woman's contraception practices and alcohol use were assessed and feedback about AEP risk was provided. The woman received informational materials about the effects of alcohol on a fetus and FASD, risky levels of alcohol use, and contraception methods; was provided an opportunity to discuss her options and possible barriers; was assisted in setting up her AEP reduction goal (if she chose AEP reduction); received a workbook with exercises; and was scheduled for a follow-up visit. (Key structural elements of the first intervention session protocol are included in Figure 2).

The workbook consisted of condensed intervention messages that included 1) self-determination/feedback about the woman's risk for AEP; 2) defining safe alcohol use during pregnancy, if attempting to become pregnant or if at-risk of unintended pregnancy; 3) recommended drinking limits if using contraception; 4) family planning/appropriate contraception use; 5) how to reduce AEP risk; 6) work- sheets for self-evaluation of importance, confidence, and readiness to use alcohol safely; 7) plans for pregnancy or contraception, 8) decisional balance regarding alcohol and contraception use; 9) goal setting, and 10) a diary to record intercourse, contraception use, and alcohol use during the subsequent four weeks. The participant was asked to read information in the workbook and educational brochures about FASD and contraception, complete exercises and the diary between visits, and bring the book to the next clinic visit to discuss with the OB/GYN. The second session protocol is tailored to the woman's choice of pregnancy planning or contraception. (The key structural elements of the second intervention session are included in Figure 3). The two DFBPI sessions were incorporated into routine OB/GYN clinic visits and could include taking a medical history, conducting a physical exam, and providing prescriptions or contraceptives if indicated. The estimated total time required for physicians to deliver the DFBPI was 5-10 min per session.

\section{Training and monitoring of physicians}

Physicians were trained in the intervention by the study supervisors who were $\mathrm{PhD}$ level psychologists and a senior $\mathrm{MD} / \mathrm{PhD} \mathrm{OB} / \mathrm{GYN}$ physician. The training included a three-hour FASD education module on the effects of alcohol consumption during pregnancy, FASD, prevention, and screening and brief interventions followed by a fourhour instructional and practice workshop. The physicians learned the DFBPI protocol, application of basic MI principles, and practiced conducting the intervention in a nonjudgmental and empathic manner. The intervention protocol was presented in a step-by-step format with instructions, and skill training techniques such as scenarios, video demonstration, and role plays, which were employed to train physicians to the behavioral competency criteria by demonstrating their delivery of all components of the 5-10 min DFBPI protocol. Feasibility and deliverability data for the DFBPI protocol were drawn from project quality control efforts. Physicians delivering DFBPI were monitored in four ways. Monitoring included reviews of the intervention fidelity check lists (see Measures section) completed by physicians and by patients after each intervention session, direct observations of physician's intervention interactions with patients conducted by the study supervisors (at least one session was observed for each physician at the beginning of the study), and reviews of audio recordings of clinic intervention visits. The OB/GYN intervention fidelity plan required completing $80 \%$ of the components of the protocol with $90 \%$ of patient contacts. The project supervising faculty, which included $\mathrm{PhD}$ psychologists, an OB/GYN, and a substance abuse physician, were available to provide feedback, consult about cases, problem-solve, and provide coaching as necessary. 


\section{Step 1: Ask}

Assess contraception/pregnancy planning and alcohol consumption:

Ask "How often have you had four or more drinks? "or use T-ACE, TWEAK, or AUDIT

Step 2: Feedback

Provide feedback and information/facts about the effects of alcohol

Step 3: Advise and help to set up an AEP prevention goal: alcohol free pregnancy or contraception

Give Advice

If there is no risk, support the woman's behavior

If there is arisk for the woman or fetus, discuss the behavior change: reducing/abstaining from alcohol and/or contraception

Assess readiness to change

Help to set upan AEP prevention goal if the woman chooses AEP reduction

If the woman is not ready for change, do not insist on setting up a goal (Ask "How would you feel if you had a child with FASD as a result of your drinking?")

Support any steps in right direction,repeat the advice, reaffirm your willingness to help when she is ready/don't move to 4

\section{Step 4: Assist}

Ask about the possible barriers and discuss how to overcome or reach the goal reaffirm your willingness to help (refer if needed)

Assist with contraception if needed

Step 5: Follow-up

Figure 1 Dual-Focused BPI protocol.

\section{Measures}

The intervention fidelity check lists (FCL) were developed for this study and included the key structural aspects of the intervention protocol. FCL were completed by women and physicians independently after each clinic intervention visit. Patient exit interviews regarding clinic visits have been used in research to monitor intervention delivery and determine feasibility of interventions [47-49]. FCL were reviewed by Russian project faculty and consultants and pilot tested prior to implementation. Women's FCL were administered in person to patients by the study research assistants immediately after the session. Examples of women's and physicians' FCL are included in Figure 2 (women's FCL for the first session) and Figure 3 (physicians' FCL for the second session). As specified by the intervention protocol, the first visit intervention components were uniform for all women while the second intervention visit components varied depending on the goals selected by the woman. The first

\begin{tabular}{|c|c|c|}
\hline Question & Yes & No \\
\hline \multicolumn{3}{|c|}{ The doctor asked me if I planned a pregnancy or used contraception. } \\
\hline \multicolumn{3}{|l|}{ The doctor asked me about my alcohol consumption. } \\
\hline \multicolumn{3}{|c|}{ The doctor told me about the incompatibility of pregnancy and alcohol use. } \\
\hline \multicolumn{3}{|c|}{ The doctor provided information about negative effects of alcohol on the fetus and child's health. } \\
\hline \multicolumn{3}{|c|}{ The doctor advised me to make a choice: either stop/reduce drinking or use effective contraception. } \\
\hline \multicolumn{3}{|l|}{ The doctor asked me what I would choose. } \\
\hline \multicolumn{3}{|c|}{ The doctor helped me to make my choice (stop/reduce drinking or use effective contraception). } \\
\hline \multicolumn{3}{|l|}{ The doctor talked to me how to achieve the goal. } \\
\hline \multicolumn{3}{|l|}{ The doctor discussed with me barriers I might face. } \\
\hline \multicolumn{3}{|l|}{ The doctor made a follow-up appointment } \\
\hline I felt the doctor's support and willingness to help me. & & \\
\hline
\end{tabular}

Figure 2 Fidelity Check List - 1st clinic visit (Women's Form). 


\begin{tabular}{|c|c|c|}
\hline Question & Yes & No \\
\hline \multicolumn{3}{|l|}{ 1. Discussed the goal that the woman chose for the prior month } \\
\hline \multicolumn{3}{|l|}{$\begin{array}{l}\text { 2. Reviewed the work book (how the woman was doing in regard to her goal of pregnancy } \\
\text { planning or contraception, successes and/or difficulties) }\end{array}$} \\
\hline \multicolumn{3}{|l|}{$\begin{array}{l}\text { 3. The woman plans pregnancy: } \\
\text { Recommended preparations for pregnancy }\end{array}$} \\
\hline \multicolumn{3}{|l|}{$\begin{array}{l}\text { 4. The woman plans pregnancy: } \\
\text { Recommended complete abstinence from alcohol }\end{array}$} \\
\hline \multicolumn{3}{|l|}{$\begin{array}{l}\text { 5. The woman plans to use contraception: } \\
\text { Recommended effective contraception }\end{array}$} \\
\hline \multicolumn{3}{|l|}{$\begin{array}{l}\text { 4. The woman plans to use contraception: } \\
\text { Recommended responsible drinking }\end{array}$} \\
\hline \multicolumn{3}{|l|}{ 5. Demonstrated support and willingness to help } \\
\hline 6. If a referral was made, specify to which specialist she was referred & & \\
\hline
\end{tabular}

Figure 3 Fidelity Check List - 2nd clinic visit (Physicians' Form).

session FCL completed by physicians and women were utilized in this implementation study.

As an additional measure to ensure that physicians maintain intervention skills over the time, audio recording of first intervention sessions was implemented in year 2 of the study. The audiotapes were coded using the FCL by two research investigators independently (85\% agreement between coders). The physician's intervention style/implementation of MI skills, including how non-confrontational/ non-judgmental the physicians' style of interaction was, how great an opportunity the patient had to set up her own goal, and how much the physician supported the patient's self-confidence were coded on a scale 1 to 5 .

\section{Data analysis}

Categorical variables were summarized as proportions. Categorical contingency tables were analyzed with McNemar's test if the data were paired and through a chi-square test if they were not. Continuous variables were summarized with means, standard deviations, and ranges. An alpha of 0.05 was considered statistically significant.

\section{Results and discussion}

FCL were completed for all first clinic intervention visits $(\mathrm{N}=374)$ conducted by 23 physicians. Two patient FCLs were incomplete, which resulted in a total of 372 patient FCL (196 in SPB and 176 in NNR) and 23 physician FCLs utilized in the analysis.

The proportions of completion of the intervention components by physicians' and women's reports are included in Figure 4.

Completion of the intervention components varied between 100\% (asked about alcohol consumption, informed about incompatibility of pregnancy and alcohol use, and helped to make a choice) and 96.5\% (discussed barriers) by physicians' reports and between 100\% (informed about incompatibility of pregnancy and alcohol use) and 93.8\% (discussed barriers) by women's reports (Figure 4). The differences between women's and physicians' reports on individual items were not significant. Physician and patient FCL reports were consistent in the majority of cases $(\mathrm{N}=307)$; however, some discrepancy existed between the reports in 57 cases (Table 1). Women reported more intervention components missing compared to physicians' self-reports $(\mathrm{p}<0.0001)$.

Similar results were received for a subset of interventions $(\mathrm{N}=78)$ by 12 physicians that were audio recoded. Out of 90 first intervention sessions completed from October 5, 2010 to July 7, 2011, 81 were audio recorded (5 patients did not consent to audio recording and 4 sessions were not recorded because of technical problems). It was not possible to code three tapes because of sound quality, resulting in a sample of 78 coded audiotapes (87\% of all sessions). In 62 cases, there was an agreement between physicians' and women's FCL reports on whether intervention components were completed; however, in 13 cases a discrepancy existed between physicians' and women's FCL reports, which indicated a significant difference between physician and women's self-reports about completed intervention components $(\mathrm{p}<0.001)$. Audiotape coding indicated that physicians implemented basic MI skills, which included nonconfrontational/nonjudgmental style of interaction (94.7\% out of an ideal $100 \%$ score), provided the patient with an opportunity to set up her own goals $(90.7 \%$ out of $100 \%$ ), and supported the patient's self-confidence ( $88 \%$ out of $100 \%$ ). Reviews of audiotapes of the intervention with patients revealed that discussing how to achieve a goal selected by a woman and discussing barriers were 
more likely to be omitted when the woman's goal was contraception. If a woman chose reducing alcohol consumption and planned pregnancy, physicians were more likely to discuss ways to reduce/avoid drinking and possible barriers. When the goal was delaying pregnancy, discussions of choices of contraception and barriers to consistent contraception use were limited or omitted.

\section{Discussion}

The overall pattern of results supports the feasibility and deliverability of a brief dual-focus AEP prevention model by OB/GYN physicians in Russia. The results are consistent with results of prior studies of alcohol reduction BPI and MI outside of Russia, namely that physicians will counsel their patients if they are provided skill training and quality control support [49,50]. Recruitment and participation agreement rates were high, and the majority of physicians who attended the training met skill criteria. Physicians trained in DFBPI and provided with support, individual feedback about their performance, coaching, and consultation during the clinical trial demonstrated high rates of delivery of all DFBPI components. They were able to implement the intervention and integrate it into routine women's clinic visits. Based on reports from physicians, patients, and audiotapes, physicians outperformed the study intervention fidelity plan requirement that was set initially at $80 \%$ of the components of the intervention with $90 \%$ of patient contacts. Similarly to Babor et al. [49], both physicians' and women's reports indicated high performance in delivery of the intervention components. However, a discrepancy between women's and physicians' reports was significant with women more likely reporting omission of specific intervention components compared to physicians' selfreports. Although there may be several explanations for the discrepancy, a review of audiotapes indicated that providers may have thought that a patient had already understood a point so they did not need to cover it much when in fact the patient did not. That was observed particularly when physicians discussed with patients contraception options and possible barriers to reducing alcohol use or utilizing contraception consistently.

Discussing difficulties/barriers that may prevent a woman from achieving her AEP prevention goal appeared to be the most difficult component for physicians to implement (or for women to grasp) and was more likely to be omitted than other components of DFBPI. In Russia, family planning and contraception counseling are conducted by OB/GYN physicians as a part of routine women's health care. Therefore, the DFBPI training was focused more on intervention components related to alcohol consumption, which is not typically a part of OB/GYN services. The assumption was that if a woman selected delaying pregnancy/

\begin{tabular}{|l|l|l|l|}
\hline & The doctor... & $\begin{array}{l}\text { Woman's report Physician's } \\
(\mathbf{N = 3 7 2})\end{array}$ & 0.997 \\
\hline 1 & asked if I planned a pregnancy or used contraception & 0.987 & 1 \\
\hline 2 & asked about my alcohol consumption & 0.989 & 1 \\
\hline 3 & $\begin{array}{l}\text { told me about the incompatibility of pregnancy and } \\
\text { alcohol use }\end{array}$ & 1 & 1 \\
\hline 4 & provided information on alcohol effects on the child & 0.995 & 0.995 \\
\hline 5 & $\begin{array}{l}\text { advised to either stop/reduce drinking or use effective } \\
\text { contraception }\end{array}$ & 0.995 & 0.995 \\
\hline 6 & asked me what I would choose & 0.949 & 0.997 \\
\hline 7 & helped me to make my choice & 0.959 & 1 \\
\hline 8 & discussed with me how to achieve the goal & 0.941 & 0.970 \\
\hline 9 & discussed barriers with me & 0.938 & 0.965 \\
\hline 10 & made a follow-up appointment & 0.978 & 0.992 \\
\hline 11 & I felt doctor's support and willingness to help & 0.997 & 0.992 \\
\hline
\end{tabular}

Figure 4 Proportion of completed intervention components. 'The differences between women's and physicians' reports on individual items were not significant. 
Table 1 Summary of physician and patient report discrepancies ${ }^{1}$

\begin{tabular}{llcc}
\hline & & \multicolumn{2}{c}{ Physician (N=23) } \\
\cline { 3 - 4 } & & NO & YES \\
\hline Patient $(\mathrm{N}=372)$ & NO & 12 & 47 \\
& YES & 10 & 295 \\
\hline
\end{tabular}

${ }^{1}$ Discrepancies between physician and women responses were summed across all FCL questions. For example, if a patient reported Yes for every $\mathrm{FCL}$ question and a physician reported Yes for every question, there was agreement, which was coded as Yes/Yes (295 cases). If a patient reported Yes for every question but her physician reported No for one or more questions, that was Yes/No (10 cases). Discrepencies between physicians and patients reports were significant $(p<0.0001)$.

contraception as her AEP reduction goal, the OB/GYN would be equipped with skills to address her goal of improving contraception use. Unexpectedly, physicians were more likely to omit discussing methods of achieving goals and possible barriers when a woman chose delaying pregnancy. The physicians trained in DFBPI and MI basic principles to address alcohol consumption appeared to be comfortable delivering alcohol reduction intervention components of the intervention; however, they demonstrated difficulty in implementing basic MI principles to address inconsistent contraception, e.g., providing information about contraception methods and discussing options and possible barriers to improve consistent contraception use. $\mathrm{OB} / \mathrm{GYN}$ physicians may benefit from expanding the contraception component of the training and developing skills to better address their patients' contraception practices.

Strengths of this study include a relatively large sample size of 374 women and a combination of different methods that included physician and women's self-reports completed shortly after sessions and live or audiotaped intervention observations were important for cross-validation of the results. Monitoring was conducted systematically and patient and provider FCL were obtained for all intervention sessions. Although audio recording was completed for a subset of interventions only, results indicated agreement between the audiotape and FCL data about completion of specific intervention components. Study limitations also should be considered. The intervention was a part of a clinical trial, and physicians and patients were provided with a level of quality control support that may be higher than what is found in routine clinical practice, which may limit generalization. The study was limited to physicians in public OB/GYN clinics and to the patients attending these clinics, so generalization to other service systems should be made cautiously. It is possible that some women with high AEP risk, such as alcohol-dependent women, do not seek $\mathrm{OB} / \mathrm{GYN}$ or prenatal care, and it is not clear how well this AEP prevention model would serve these women. However, Russian government statistics indicate that $96.4 \%$ of women receive prenatal services from public women's clinics [51], and therefore, the study sample represents the major OB/GYN service delivery system in Russia.

\section{Conclusions}

This study supported the feasibility of incorporating DFBPI in routine women's clinics visits in Russia. Physicians trained in DFBPI were able to implement and maintain the intervention skills. Despite some discrepancies between women and physicians' reports regarding completed intervention components, there were far more congruencies, and $\mathrm{OB} / \mathrm{GYN}$ physicians trained in the DFBPI, monitored, and supported during the study demonstrated performance that met or exceeded benchmarks. Broader implementation of these types of clinic-based, motivational dual-focus models outside of a research context may benefit from additional research that would determine the amount and type of quality control effort needed to obtain the highest costbenefit. This study demonstrates that multisite implementation is feasible, but it does not establish an implementation strategy that is necessarily the most efficient. Research is needed to identify efficient ways to support implementation of AEP prevention interventions in clinical settings.

\section{Endnotes}

${ }^{a}$ Risk for AEP among non-pregnant women was defined as at-risk alcohol consumption (four or more drinks on one occasion or eight or more drinks per week) plus the chance or intent to become pregnant [38].

\section{Abbreviations \\ AEP: Alcohol-exposed pregnancy (pregnancies); BI: Brief intervention; BPI: Brief physician intervention; DFBPI: Dual-focused brief physician intervention; FAS: Fetal Alcohol Syndrome; FASD: Fetal Alcohol Spectrum Disorders; FLC: Fidelity check list; MI: Motivational interviewing; NNR: The Nizhny Novgorod Region, Russia; OB/GYN: Obstetrics and gynecology (obstetrics and genecology physicians); SPB: St. Petersburg, Russia.}

\section{Competing interests}

The study was supported by Grant Number R01AA016234 from the National Institutes of Health/National Institute on Alcohol Abuse and Alcoholism and Fogarty International Center and its contents are solely the responsibility of the authors and do not necessarily represent the official views of the $\mathrm{NIH}$.

The authors have no competing interests.

\section{Authors' contributions}

All authors have made substantial contributions to conception, design, gathering data, analysis, and/or interpretation of data and have contributed to the intellectual content and writing of the article. Specifically, TB: is PI on the study and led development of the study design, the intervention protocol and materials, measures, conducted supervision, coding, participated in data analysis, and drafted the manuscript; BB: have significantly contributed to the study conception and coordination, design, and analyzing data; MC: provided significant contribution to the study design and conceptualization, participated in the manuscript writing; Gl: conducted research supervision, contributed significantly to developing the study materials; VS: coordinated study and collaborated with clinics, supervised and consulted physicians, conducted training of physicians; LT: contributed to the study conceptualization and coordination; EV: conducted research supervision, contributes to developing the study materials, provided significant contribution to the study coordination; IG: coordinated clinics and conducting interventions, conducted training of physicians and supervision; LS: coordinated data collection and conducting interventions, participated in 
physicians supervision, prepared data for analysis; NK: participated in designing the study, performing statistical analysis, and preparing the manuscript. All authors read and approved the final manuscript.

\section{Acknowledgments}

Authors wish to thank consultants Linda Sobell, PhD, of Nova Southeastern University and Michael Fleming, MD/MPH, of the University of WisconsinMadison (currently at Northwestern University) for their invaluable advise to the study and Karen Beckman, MD, of the University of Oklahoma Health Science Center, the study advisor on protection of human subjects in research, acknowledge contribution of data collectors - graduate students from St. Petersburg State University and Nizhny Novgorod State Pedagogical University and physician interventionists from OB/GYN clinics, and to thank the study participants.

The study was supported by Grant Number R01AA016234 from the National Institutes of Health/National Institute on Alcohol Abuse and Alcoholism and Fogarty International Center and its contents are solely the responsibility of the authors and do not necessarily represent the official views of the $\mathrm{NIH}$.

\section{Author details}

${ }^{1}$ The University of Oklahoma Health Sciences Center, 940 N.E. 13th Street, Nicholson Tower Suite 4900, Oklahoma City, OK 73104, USA. ${ }^{2}$ St. Petersburg State University, 6 Makarova nab, St. Petersburg 199053, Russia. ${ }^{3}$ St. Petersburg State Pediatric Academy, 54, Malaya Balkanskaya Str., St. Petersburg 192283, Russia. ${ }^{4}$ Nizhny Novgorod State Pedagogical University, 12 UlyanovaStr., Nizhniy Novgorod 603950, Russia. ${ }^{5}$ NSK Statistical Solutions LLC, Choctaw, OK 73020, USA.

Received: 13 March 2012 Accepted: 14 December 2012

Published: 8 January 2013

\section{References}

1. American Academy of Pediatrics, Committee on Substance Abuse and Committee on Children With Disabilities: Fetal alcohol syndrome and alcohol-related neurodevelopmental disorders. Pediatrics 2000, 106:358-361

2. Bertrand J, Floyd RL, Weber MK, O'Connor M, Riley EP, Johnson KA, Cohen DE: National Task Force on FAS/FAE. Fetal Alcohol Syndrome: Guidelines for Referral and Diagnosis. Atlanta: Centers for Disease Control and Prevention; 2004.

3. Streissguth AP, O'Malley K: Neuropsychiatric implications and long-term consequences of fetal alcohol spectrum disorders. Semin Clin Neuropsychiatry 2000, 5(3):177-190.

4. Warren KR, Hewitt BG, Thomas JD: Fetal alcohol spectrum disorders: research challenges and opportunities. Alcohol Res Health 2011, 34(1):4-14

5. Abel EL, Sokol RJ: Incidence of fetal alcohol syndrome and economic impact of FAS-related anomalies. Drug Alcohol Depend 1987, 19:51-70.

6. May PA, Gossage JP, Kalberg WO, Robinson LK, Buckley D, Manning M, Hoyme HE: Prevalence and epidemiologic characteristics of FASD from various research methods with an emphasis on recent in-school studies. Dev Disabil Res Rev 2009, 15(3):176-192.

7. Miller LC, Chan W, Litvinova A, Rubin A, Comfort K, Tirella L, Cermak S, Morse B, Kovalev I: Fetal alcohol spectrum disorders in children residing in Russian orphanages: a phenotypic survey. Alcohol Clin Exp Res 2006, 30:531-538.

8. Riley EP, Guerri C, Calhoun F, Charness ME, Foroud TM, Li TK, Mattson SN, May PA, Warren KR: Prenatal alcohol exposure: advancing knowledge through international collaborations. Alcohol Clin Exp Res 2003, 27:118-135.

9. Palchik A, Legonkova S, Sofronova G: Этнические особенности проявления алкогольного синдрома плода [Ethnic features of fetal alcohol syndrome manifestation]. Yakut Med J 2011, 34:22-25. (In Russian). http://ymj.ykt.ru/images/stories/2-2011.pdf.

10. Landgren M, Svensson L, Strömland K, Andersson Grönlund M: Prenatal alcohol exposure and neurodevelopmental disorders in children adopted from Eastern Europe. Pediatrics 2010, 125:e1178-e1185.

11. Floyd RL, O'Connor MJ, Sokol RJ, Bertrand J, Cordero JF: Recognition and prevention of fetal alcohol syndrome. Obstet Gynecol 2005, 106:1059-1064.

12. Warren KR, Foudin LL: Alcohol-related birth defects-the past, present, and future. Alcohol Res Health 2001, 25(3):153-158.

13. Centers for Diseases Control and Prevention: Alcohol use among pregnant and nonpregnant women of childbearing age: United States, 1991-2005. MMWR Morb Mortal Wkly Rep 2009, 58(19):529-532.

14. Skagerstrom J, Chang G, Nilsen P: Predictors of drinking during pregnancy: a systematic review. J Womens Health 2011, 20:901-913.
15. Floyd RL, Decoufle P, Hungerford DW: Alcohol use prior to pregnancy recognition. Am J Prev Med 1999, 17:101-107.

16. Ethen MK, Ramadhani TA, Scheherele AE, Canfield MA, Wyszynsji DF, Druschel CM, Romitti PA: National birth defects prevention study. Matern Child Health J 2007, 13:274-285.

17. Clarren SK, Astley SJ, Gunderson VM, Spellman D: Cognitive and behavioral deficits in nonhuman primates associated with very early embryonic binge exposures to ethanol. J Pediatr 1992, 121(5 Pt 1):789-796.

18. Floyd RL, Ebrahim SH, Boyle CA, Observations from the CDC: Preventing alcohol-exposed pregnancies among women of childbearing age: the necessity of a preconceptional approach. J Womens Health Gend Based Med 1999, 8:733-736.

19. Balachova T, Bonner B, Chaffin M, Bard D, Isurina G, Tsvetkova L, Volkova E: Women's alcohol consumption and risk for alcohol-exposed pregnancies in Russia. Addiction 2012, 107(1):109-117.

20. Kristjanson AF, Wilsnack SC, Zvartau E, Tsoy M, Novikov B: Alcohol use in pregnant and non-pregnant Russian women. Alcohol ClinExp Res 2007, 31:299-307.

21. Chambers CD, Kavteladze L, Joutchenko L, Bakhireva LN, Jones KL: Alcohol consumption patterns among pregnant women in the Moscow region of the Russian Federation. Alcohol 2006, 38:133-137.

22. The Project CHOICES Intervention Research Group: Reducing the risk of alcohol-exposed pregnancies: a study of a motivational intervention in community settings. Pediatrics 2003, 111(5):1131-1135.

23. Babor TF, McRee BG, Kassebaum PA, Grimaldi PL, Ahmed K, Bray J: Screening, brief intervention, and referral to treatment (SBIRT): toward a public health approach to the management of substance abuse. Subst Abus 2007, 8(3):7-30.

24. Whitlock EP, Polen MR, Green CA, Orleans T, Klein J: Behavioral counseling interventions in primary care to reduce risky/harmful alcohol use by adults: a summary of the evidence for the U.S. preventive services task force. Ann Intern Med 2004, 140(7):557-568.

25. Chang G, Mcnamara TK, Orav EJ: Brief intervention for prenatal alcohol use: a randomized trial. Obstet Gynecol 2005, 105(5):991-998.

26. Hankin J, McCaul ME, Heussner J: Pregnant, alcohol-abusing women. Alcohol Clin Exp Res 2000, 24:1276-1286.

27. Chang G, Goetz MA, Wilkins-Haug L, Berman S, Hankin J, McCaul ME, Heussner J: A brief intervention for prenatal alcohol use: an in-depth look. J Subst Abuse Treat 2000, 18(4):365-369.

28. Manwell LG, Fleming MF, Mundt MP, Stauffacher EA, Barry KL: Treatment of problem alcohol use in women of childbearing age: results of a brief intervention trial. Alcohol Clin Exp Res 2000, 24:1517-1524.

29. O'Connor MJ, Whaley SE: Alcohol use in pregnant low-income women. J Stud Alcohol 2003, 64(6):773-783.

30. Fleming MF, Lund MR, Wilton G, Landry M, Scheets D: The Healthy Moms study: the efficacy of brief alcohol intervention in postpartum women. Alcohol Clin Exp Res 2008, 32(9):1600-1606.

31. Nilsen P: Brief alcohol intervention to prevent drinking during pregnancy: an overview of research findings. Curr Opin Obstet Gynecol 2009, 21(6):496-500.

32. Chang G: Screening and prevention in prenatal care settings. Alcohol Res Health 2004, 28(2):80-84.

33. Committee opinion No. 496. American College of Obstetricians and Gynecologists: At-risk drinking and alcohol dependence: obstetric and gynecologic implications. Obstet Gynecol 2011, 118:383-388.

34. Stade BC, Dzendoletas, Sgro M, Dowswell T, Bennett D: Psychological and/ or educational interventions for reducing alcohol consumption in pregnant women and women planning pregnancy (Review). Cochrane Database Syst Rev 2009, 27

35. Miller WR, Sorensen JL, Selzer JA, Brigham GS: Disseminating evidencebased practices in substance abuse treatment: a review with suggestions. J Subst Abuse Treat 2006, 31(1):25-39.

36. Chang G: FASD: Prevention and nutrition: Commentary on Floyd, Denny and Weber, and Nguyen and Thomas. In Encyclopedia on Early Childhood Development [online]. Edited by Tremblay RE, Boivin M, De V, Peters R. Montreal: Centre of Excellence for Early Childhood Development and Strategic Knowledge Cluster on Early Child Development; 2011:1-5.

37. Andréasson S, Hjalmarsson K, Rehnman C: Implementation and dissemination of methods for prevention of alcohol problems in primary health care: a feasibility study. Alcohol Alcohol 2000, 35(5):525-530.

38. Babor TE, Higgins-Biddle J, Dauser D, Higgins P, Burleson JA: Alcohol screening and brief intervention in primary care settings: implementation models and predictors. J Stud Alcohol 2005, 66(3):361. 
39. Mengel MB, Searight HR, Cook K: Preventing alcohol-exposed pregnancies. J Am Board Fam Med 2006, 19:494-505.

40. Babor TF, Higgins-Biddle JC, Saunders JB, Monteiro M: AUDIT - the Alcohol Use Disorder Identification Test: Guidelines for use in Primary Health Care. 2nd edition. Geneva, Switzerland: World Health Organization, Department of Mental Health and Substance Abuse; 2001. WHO/MSD/MSB/01.6a.

41. Floyd RL, Sobell M, Velasquez MM, Ingersoll K, Nettleman M, Sobell L, Mullen PD, Ceperich S, Von Sternberg K, Bolton B, Skarpness B, Nagaraja J: Preventing alcohol-exposed pregnancies: a randomized controlled trial. Am J Prev Med 2007, 32:1-10.

42. Balachova TN, Bonner BL, Isurina GL, Tsvetkova LA: Use of focus groups in developing FAS/FASD prevention in Russia. Subst Use Misuse 2007, 42:881-894.

43. Babor TF, Higgins-Biddle JC: Brief Intervention for Hazardous and Harmful Drinking: A Manual for Use in Primary Care. Geneva: WHO; 2001.

44. Miller W, Rollnick S: Motivational Interviewing: Preparing People for Change. 2nd edition. New York: Guilford Press; 2002.

45. Balachova T, Bonner B, Isurina G, Palchik A, Shapkaitz V, Tsvetkova L: Changing physicians' knowledge, skills and attitudes to prevent FASD in Russia. Proceedings of the 33rd Annual Scientific Meeting of the Research Society on Alcoholism, Alcoholism: Clinical \& Experimental Research 2010, 34(Suppl 6):210A.

46. Shapkaitz V, Balachova TN, Regentova A: Kaк выбрать контрачеnтив [How to choose a contraception method]. St. Petersburg: SPSU-OUHSCNNSPU; 2009 (in Russian).

47. Pbert L, Fletcher K, Flint A, Young M, Druker S, DiFranza J: Smoking prevention and cessation intervention delivery by pediatric providers, as assessed with patient exit interviews. Pediatrics 2006, 118:e810-e824.

48. Franco LM, Franco C, Kumwenda N, Nkhoma W: Methods for assessing quality of provider performance in developing countries. Int I Qual Health Care 2002, 14(Suppl 1):17-24.

49. Babor TF, Higgins-Biddle JC, Higgins PS, Gassman RA, Gould BE: Training medical providers to conduct alcohol screening and brief interventions. Subst Abus 2004, 25(1):17-26.

50. D'Onofrio G, Nadel ES, Degutis LC, Sullivan LM, Casper K, Bernstein E, Samet $\mathrm{JH}$ : Improving emergency medicine residents' approach to patients with alcohol problems: a controlled educational trial. Ann Emerg Med 2002, 40(1):50-62.

51. Sukhanova LP: Статистика родовспоможения как фактор обеспечения качества акушерской и перинатальной помощи в России. [Statistics of obstetric aid as a factor of providing quality of obstetric and perinatal care in Russia]. Inf Anal Rep Soc Aspects Public Health 2008, (in Russian) http://vestnik.mednet.ru/content/view/47/30/.

doi:10.1186/1940-0640-8-1

Cite this article as: Balachova et al:: Brief FASD prevention intervention: physicians' skills demonstrated in a clinical trial in Russia. Addiction Science \& Clinical Practice 2013 8:1.

\section{Submit your next manuscript to BioMed Central and take full advantage of:}

- Convenient online submission

- Thorough peer review

- No space constraints or color figure charges

- Immediate publication on acceptance

- Inclusion in PubMed, CAS, Scopus and Google Scholar

- Research which is freely available for redistribution 